\title{
Criminologie
}

\section{Adolescents en centre de réadaptation : évaluation du trauma}

\section{Delphine Collin-Vézina et Lise Milne}

Volume 47, numéro 1, printemps 2014

La polyvictimisation des jeunes

URI : https://id.erudit.org/iderudit/1024014ar

DOI : https://doi.org/10.7202/1024014ar

Aller au sommaire du numéro

\section{Éditeur(s)}

Les Presses de l’Université de Montréal

ISSN

0316-0041 (imprimé)

1492-1367 (numérique)

Découvrir la revue

\section{Citer cet article}

Collin-Vézina, D. \& Milne, L. (2014). Adolescents en centre de réadaptation : évaluation du trauma. Criminologie, 47(1), 213-245.

https://doi.org/10.7202/1024014ar

\section{Résumé de l'article}

Considérant que les jeunes placés en centre de réadaptation ont souvent vécu des événements traumatiques multiples et que les services spécialisés en trauma ne sont pas systématiquement implantés en protection de la jeunesse, il serait souhaitable qu'une évaluation standardisée des événements et des séquelles traumatiques soit mise en place auprès de cette clientèle à risque. L'objectif de cet article est d'offrir une synthèse des outils psychométriques pour évaluer le trauma chez les jeunes, et ce, dans le but de rendre plus accessible cette information nécessaire au développement d'un programme solide et cohésif de santé mentale axé sur le traumatisme en protection de la jeunesse. Sur la base d'une démarche systématique, 13 outils qui visent les événements et les symptômes traumatiques ont été sélectionnés, la plupart étant des questionnaires que les jeunes remplissent et des entrevues dirigées. Les considérations pratiques et méthodologiques de ces outils sont présentées. L'implantation d'une évaluation systématique des traumas pour les enfants et les jeunes en centre de réadaptation pourrait servir de tremplin à la mise sur pied de services spécialisés pour cette clientèle dont les besoins sont criants. Les interventions les plus prometteuses sont discutées. 


\title{
Adolescents en centre de réadaptation : évaluation du trauma
}

\author{
Delphine Collin-Vézina ${ }^{1}$ \\ Chaire de recherche junior du Canada en aide sociale à l'enfance \\ Université McGill \\ delphine.collin-vezina@mcgill.ca \\ Lise Milne \\ Doctorante \\ Université McGill \\ lise.milne@mcgill.ca
}

RÉSUMÉ • Considérant que les jeunes placés en centre de réadaptation ont souvent vécu des événements traumatiques multiples et que les services spécialisés en trauma ne sont pas systématiquement implantés en protection de la jeunesse, il serait souhaitable qu'une évaluation standardisée des événements et des séquelles traumatiques soit mise en place auprès de cette clientèle à risque. L'objectif de cet article est d'offrir une synthèse des outils psychométriques pour évaluer le trauma chez les jeunes, et ce, dans le but de rendre plus accessible cette information nécessaire au développement d'un programme solide et cohésif de santé mentale axé sur le traumatisme en protection de la jeunesse. Sur la base d'une démarche systématique, 13 outils qui visent les événements et les symptômes traumatiques ont été sélectionnés, la plupart étant des questionnaires que les jeunes remplissent et des entrevues dirigées. Les considérations pratiques et méthodologiques de ces outils sont présentées. L'implantation d'une évaluation systématique des traumas pour les enfants et les jeunes en centre de réadaptation pourrait servir de tremplin à la mise sur pied de services spécialisés pour cette clientèle dont les besoins sont criants. Les interventions les plus prometteuses sont discutées.

MOTS-CLÉS - Trauma, évaluation, centres de réadaptation, adolescents.

\section{Introduction}

Lorsque la sécurité ou le développement d'un adolescent est compromis dans son milieu de vie, ou lorsqu'il présente un risque sérieux de danger

1. Université McGill, Wilson Hall, École de service social, 3506, rue University, Montréal, (Québec), Canada, H3A 2A7. 
pour lui-même ou pour autrui, un placement dans un centre de réadaptation (CR) peut s'avérer nécessaire. Le placement en CR constitue la forme la plus intensive d'intervention qui, la plupart du temps, fait suite à une ordonnance de la Cour. Ce placement est effectué uniquement lorsque d'autres mesures moins extrêmes ont échoué à résoudre les problèmes ayant nécessité l'intervention de la protection de la jeunesse, que ce soit pour des motifs liés à la Loi de la protection de la jeunesse et/ ou à la Loi sur le système de justice pénale des adolescents. Une analyse de 4120 enfants et adolescents québécois placés démontre que plus de la moitié d'entre eux $(52,5 \%)$ se retrouve en CR à un moment de leur trajectoire de services (Hélie et al., soumis). En outre, cette analyse démontre que la majorité $(60 \%)$ des jeunes qui sont placés en CR au Québec sous la Loi de la protection de la jeunesse le sont pour un motif de protection qui n'existe pas ailleurs: les troubles de comportement sérieux, soit «lorsque l'enfant, de façon grave ou continue, se comporte de manière à porter atteinte à son intégrité physique ou psychologique ou à celle d'autrui, et que ses parents ne prennent pas les moyens nécessaires pour mettre fin à la situation ou que l'enfant de 14 ans et plus s'y oppose» (article $38 \mathrm{f}$ ). En d'autres termes, lorsque la situation devient trop difficile dans le milieu de vie actuel d'un jeune, le placement en hébergement peut être recommandé. Dans ce contexte, des services de réadaptation, souvent basés sur l'apprentissage des habiletés sociales, lui seront offerts en plus de la scolarisation afin de corriger les comportements à risque, et ce, dans l'espoir de lui permettre de réintégrer son milieu de vie.

Or, les problèmes de ces jeunes ne se limitent pas aux motifs de placement actuels ciblés par les services en CR. Outre leurs problèmes de comportement, on reconnaît de plus en plus que les adolescents placés par la protection de la jeunesse présentent des problèmes aigus de santé mentale, notamment des épisodes de dépression majeure, des troubles de l'attention, des comportements sexualisés inadéquats, de la consommation abusive de substances, de l'agressivité et des idées suicidaires (McMillen et al., 2005; Baker et al., 2007; Egelund \& Lausten, 2009; Guibord et al., 2011). Jusqu'à $82 \%$ de ces jeunes présentent les critères d'au moins un trouble majeur répertorié dans le DSM-IV (Trout et al., 2008) et au moins un tiers d'entre eux ont connu une hospitalisation psychiatrique (Dale et al., 2007). En outre, les symptômes d'anxiété, la dépression et les troubles de l'attention sont signalés de façon plus importante chez les adolescents placés dans des CR que chez ceux 
qui reçoivent des services de la protection de l'enfance, mais à leur domicile (Tarren-Sweeney, 2008; Kolko et al., 2010). Dans l'une des seules études canadiennes sur ce sujet (Guibord et al., 2011), les données recueillies auprès de 122 jeunes Ontariens et Ontariennes en situation de placement indiquent que $40 \%$ des participants ont éprouvé au moins un des deux problèmes de santé mentale suivants au cours de l'année: la dépression et la consommation abusive de substances.

Trois facteurs doivent être considérés dans l'analyse des problèmes de santé mentale chez les enfants et adolescents placés en CR: les événements traumatiques multiples qui ont été vécus par une majorité de ces jeunes, une offre limitée de services spécialisés en trauma dans les unités, et le manque d'évaluation standardisée des traumatismes et des séquelles qui en découlent dans les CR.

\section{Événements traumatiques multiples}

Des années de connaissances cliniques ont influencé le déploiement récent de recherches portant sur les traumatismes vécus par les jeunes en CR, ce qui a permis de confirmer que les troubles émotionnels et comportementaux manifestés par ceux-ci pouvaient représenter, du moins en partie, des symptômes liés à un cumul d'expériences de vie traumatisantes (Cook et al., 2005). Les traumatismes vécus par les jeunes en CR sont aujourd'hui bien documentés, et incluent l'abandon des parents (chez $34 \%$ à $42 \%$ des jeunes), la négligence physique ou émotionnelle $(50 \%$ à $98 \%)$, la violence physique (40\% à $81 \%)$, les agressions sexuelles (12\% à $54 \%$ ) et l'exposition à la violence conjugale (65\% à $85 \%$ ) (Brady \& Caraway, 2002; Hussey \& Guo, 2002; Baker et al., 2003 ; Connor et al., 2004; Baker et al., 2006; Dale et al., 2007; Griffin et al., 2009; Arvidson et al., 2011; Dorsey et al., 2012). Les recherches démontrent que ces jeunes vivent plus fréquemment l'expérience de traumatismes multiples, plutôt que de subir un incident de traumatisme isolé, ce qui entraîne des perturbations graves et affligeantes. Par exemple, Brady et Caraway (2002) ont constaté que la majorité $(65,8 \%)$ de leur échantillon de jeunes en CR avait connu trois types de traumatismes ou plus. De même, une étude de Baker, Curtis et Papa-Lentini (2006) rapporte qu'un peu plus de 36\% des jeunes vivant dans les $C R$ avaient été victimes d'une combinaison de négligence, de mauvais traitements physiques et d'agressions sexuelles. Griffin et ses collègues (2011) ont mené une étude de grande envergure 
avec plus de 14000 enfants âgés entre 0 et 17 en situation de placement, afin d'évaluer leurs expériences traumatiques, les symptômes liés au traumatisme, et la relation entre leurs expériences et leurs symptômes. Les résultats ont démontré que, dans $75 \%$ des cas, les enfants avaient vécu au moins un événement traumatisant considéré modéré à sévère. Au total, plus d'un quart des enfants avaient des symptômes traumatiques de niveau clinique. En outre, le nombre moyen de symptômes de traumatisme était directement lié au nombre d'événements traumatiques vécus. Ces résultats trouvent écho dans une étude québécoise menée auprès d'un échantillon de 53 adolescents et adolescentes de 14 à 17 ans provenant de six unités de réadaptation. En effet, $38 \%$ de l'échantillon a rapporté avoir été agressé sexuellement, $58 \%$ a été émotionnellement négligé, $60 \%$ a été maltraité physiquement, $68 \%$ a souffert de violence psychologique, et près de la totalité de l'échantillon a souffert de négligence physique (Collin-Vézina et al., 2011). Plus de la moitié de l'échantillon avait connu quatre ou cinq différentes formes de traumatisme sur cinq $(51 \%)$. Le quart de ce groupe de jeunes présentait des symptômes cliniques de stress post-traumatique, de dissociation et de dépression. Pourtant, seule une minorité de ces jeunes étaient pris en charge en raison des expériences de traumatismes. En effet, $83 \%$ d'entre eux étaient placés pour des problèmes de comportement et non des expériences de maltraitance (Milne \& Collin-Vézina, sous presse). Récemment, on a également noté auprès de jeunes en $\mathrm{CR}$ la présence fréquente de traumatismes vécus dans la communauté, et non uniquement dans leur milieu familial (Arvidson et al., 2011; Dorsey et al., 2012). Cette affirmation est également confirmée dans une étude québécoise auprès de 220 jeunes pris en charge par la protection de la jeunesse (Cyr et al., 2012) où le taux de traumatismes multiples, ou polyvictimisation (quatre expériences de victimisation vécues dans les différents milieux de vie de l'enfant), était de $54 \%$, en comparaison de seulement $8 \%$ dans un échantillon représentatif d'enfants et d'adolescents québécois (Cyr et al., 2013).

Selon Brière et Lanktree (2008), les effets des traumatismes multiples et complexes se manifestent bien au-delà du trouble de stress posttraumatique et se révèlent à travers une multitude de troubles de santé mentale, notamment l'anxiété et la dépression, la dissociation, les problèmes de régulation émotionnelle et relationnelle, les distorsions cognitives, la somatisation, l'automutilation, les perturbations sexuelles, la toxicomanie et les troubles alimentaires. En bref, ces multiples expé- 
riences de traumatisme entraînent des perturbations émotionnelles et comportementales graves (Baker et al., 2006), qui se manifestent par des déficits sur le plan de l'attachement, de la régulation des affects et du développement de compétences (Blaustein \& Kinniburgh, 2010). Ces difficultés risquent de sérieusement compromettre le parcours développemental des jeunes (D’Andrea et al., 2012). Les comportements déviants et inadaptés qui ont précipité le placement en CR des jeunes pourraient ainsi être interprétés comme des adaptations dysfonctionnelles liées à des expériences traumatiques et, par conséquent, être le reflet ou le symptôme d'enjeux plus profonds qui se doivent d'être investigués et traités (Runtz \& Briere, 1986; Singer et al., 1995).

\section{Accès aux services spécialisés en trauma}

Il est bien documenté que les enfants et les adolescents en situation de placement sont de grands utilisateurs de médications psychotropes. Par exemple, parmi un échantillon de jeunes en CR, $82 \%$ d'entre eux avaient reçu une prescription de médicaments psychotropes au moment de leur admission (Baker et al., 2007), une réalité confirmée dans une étude québécoise (Lafortune, 2006). Toutefois, la capacité des CR de répondre aux besoins complexes et variés de cette population, au-delà des prescriptions de médicaments psychotropes et des interventions axées sur les habiletés sociales, est remise en question (Burns et al., 2004; Petrenko et al., 2011). Par exemple, malgré des difficultés psychologiques cliniquement élevées dans un échantillon de 292 enfants et jeunes en placement, seulement $29 \%$ recevaient des services spécialisés en santé mentale (Jannsens \& Deboutte, 2009). En fait, les services offerts à ces enfants sont susceptibles de varier d'un CR à l'autre, puisqu'aucun modèle commun d'intervention ciblée ne constitue la base des services en CR qui sont, le plus souvent, tout simplement étiquetés comme une "communauté thérapeutique» ou «cognitivo-comportementale» (Betterman \& Jasperson, 2009). Ceci étant dit, les recherches qui témoignent d'un manque de services en santé mentale ont été menées dans des contextes autres que celui du Québec; des pratiques prometteuses sont déjà en place à travers différents Centres jeunesse, notamment des unités de CR en santé mentale. Il est toutefois probable que ces pratiques ne soient pas uniformisées à travers les 16 Centres jeunesse du Québec et qu'elles ne visent pas le trauma plus particulièrement. Au Québec, bien que des formations de 
base soient offertes aux intervenants œuvrant en protection de l'enfance par l'entremise du Programme national de formation jeunesse (PNFJ), il n'y a pas d'approche systématique pour développer des services axés sur le traumatisme et basés sur des données probantes laissant, par conséquent, le soin à chaque agence de promouvoir et d'implanter des programmes jugés pertinents pour leur clientèle. Une réorganisation des services en CR autour de la question des traumatismes et des séquelles découlant de ces événements adverses est recommandée par de nombreux experts (Briere \& Lanktree, 2008; Blaustein \& Kinniburgh, 2010; Hummer et al., 2010). À cet effet, selon le National Child Trauma Stress Network (NCTSN), leader mondial dans le domaine des traumas multiples et complexes, le manque de services spécialisés et basés sur des données probantes est criant pour traiter les séquelles traumatiques des jeunes desservis par la protection de la jeunesse (Taylor \& Siegfried, 2005). Le peu de connaissances actuelles concernant l'efficacité des interventions en $\mathrm{CR}$ serait également le reflet du manque d'études scientifiques menées dans ces milieux d'intervention, ce qui démontre l'importance de mettre en place des recherches évaluatives rigoureuses (Walter, 2007). Ces recherches devraient prendre racine dans une évaluation rigoureuse des événements traumatiques et de leurs séquelles, ce qui constitue un champ d'expertise fondamental pour la mise en place de programmes d'intervention pertinents pour cette clientèle.

\section{Évaluation standardisée des traumas et de leurs séquelles}

Dans cette foulée, de nombreux chercheurs ont souligné l'importance d'une évaluation systématique des besoins des enfants et des jeunes traumatisés afin d'apporter un portrait éclairé des besoins de cette clientèle (Greenwald, 1999; Strand et al., 2005; Igelman et al., 2007; Nader, 2008). Plus particulièrement, ceux-ci soulignent l'importance de la mise en place d'une telle évaluation afin d'offrir des services qui répondent à l'ensemble de la présentation clinique des enfants et des jeunes, et non uniquement aux troubles de comportement (Carter et al., 2004; Strand et al., 2005). À cet effet, le NCTSN recommande aux services de la protection de la jeunesse l'utilisation d'outils standardisés adaptés à l'âge de l'enfant et qui couvrent différentes facettes de son histoire et de son développement (NCTSN, 2008). Ces outils porteraient une attention particulière aux événements traumatiques afin de 
contextualiser les problèmes émotionnels et comportementaux actuels à la lumière des déficits engendrés par le cumul d'adversités vécues (Taylor \& Siegfried, 2005). Cette évaluation devrait, notamment, être utilisée pour la prise de décision clinique et être discutée avec les membres de la famille, les tribunaux et les équipes multidisciplinaires (Taylor \& Siegfried, 2005). Ces recommandations sont partagées par d'autres leaders dans le domaine du trauma, notamment Briere et Lanktree (2008), qui insistent également sur la nécessité d'effectuer ces évaluations à intervalles réguliers afin de documenter l'évolution positive ou la détérioration de l'état de santé mentale des jeunes en CR, et d'inclure des mesures de fonctionnement des enfants et des jeunes, notamment au sujet de l'attachement, de la régulation affective et comportementale, du développement de l'identité et des stratégies d'adaptation.

Malgré une plus grande reconnaissance de l'histoire de traumatismes multiples vécus par les jeunes en CR, force est de constater l'absence fréquente d'évaluation standardisée des troubles de santé mentale et des événements traumatiques lors de l'admission des enfants et des jeunes en CR (Mash \& Hunsley, 2005). Certaines instances gouvernementales ont récemment émis des recommandations claires à cet effet, notamment le Congrès américain qui exige désormais des services de protection de l'enfance une réponse soutenue et concertée pour répondre aux besoins des enfants et des jeunes en situation de placement (Stoltzfus, 2011). De même, l'American Academy of Child and Adolescent Psychiatry et la Child Welfare League of America (2002) ont publié une déclaration de politiques communes appelant à l'évaluation globale des questions liées au trauma, à la santé mentale et à la toxicomanie de tous les enfants en famille d'accueil. Cet énoncé est soutenu par des chercheurs, tels que McMillen et al. (2005), qui affirment que les taux élevés de troubles psychiatriques chez les jeunes en situation de placement nécessitent un dépistage systématique. Certains chercheurs notent toutefois que l'accent devrait être mis sur les évaluations réfléchies et individualisées qui influencent directement la planification du traitement et, qu'en ce sens, les protocoles d'évaluation devraient être appliqués avec discernement (Chaffin, 2000; Nader, 2008).

À la lumière de ces constats, le présent article vise à offrir une synthèse des outils psychométriques pour évaluer les événements et les séquelles traumatiques chez les jeunes en CR. L'implantation d'une approche systématique d'évaluation pourrait constituer une étape 
importante vers la transformation des services traditionnels de protection de l'enfance en un programme solide et cohésif de santé mentale axé sur le traumatisme. Il nous apparaît également qu'une telle revue est inexistante en français, une lacune que cette recension espère combler.

\section{Outils d'évaluation pour le trauma}

Le domaine de l'évaluation du trauma est en pleine expansion, ce qui se reflète dans le nombre grandissant d'instruments pour les enfants et les jeunes victimes de traumatismes. Alors que les récentes recensions incluaient plusieurs dizaines d'instruments différents (Strand et al., 2005; Fletcher, 2007; Nader, 2008), une revue de 2002 n'en listait que 15 (Ohan et al., 2002), ce qui témoigne de l'intérêt actuel pour ce domaine d'expertise. La liste de mesures présentée ici est basée sur une recherche en trois volets. D'abord, les plus récents ouvrages concernant les mesures destinées aux jeunes (Frick et al., 2009), les mesures plus spécifiques au trauma (Ohan, et al., 2002; Strand et al., 2005; Nader, 2008; Conradi et al., 2011), et la littérature sur les enfants et les adolescents victimes de traumatismes (Briere \& Lanktree, 2008; Steele \& Malchiodi, 2012) ont été consultés pour cerner les outils psychométriques les plus reconnus par les experts dans le domaine. Ensuite, afin d'évaluer la qualité des mesures suggérées par les experts, une recension des études empiriques portant sur l'évaluation des traumas chez les enfants et les jeunes publiés depuis les années 2000 a été menée à partir des banques de données Psycinfo et Medline avec les mots-clés: child, adolescent, trauma, assessment. Finalement, afin de choisir des mesures pertinentes pour le contexte des CR, la littérature plus spécifique de la protection de la jeunesse a été analysée par l'entremise d'une revue systématique menée par le Centre de recherche sur l'enfance et la famille de l'Université McGill où, chaque mois depuis 2007, une liste à jour de publications provenant de dizaines de revues réputées est soumise à un comité de lecture dont font partie les auteures de cet article. Seuls des questionnaires et des entrevues structurées ont été retenus dans la présente démarche, car ils sont considérés comme utiles pour offrir des évaluations rapides, précises et fiables du fonctionnement et, en ce sens, sont considérés comme plus efficaces que les autres méthodes, notamment les entrevues cliniques (Strand et al., 2005). Les outils n'ayant fait l'objet d'aucune évaluation auprès de populations 
adolescentes n'ont pas été retenus, malgré leur pertinence démontrée auprès d'enfants placés d'âge préscolaire ou scolaire (par ex.: Assessment Checklist for Children; Tarren-Sweeney, 2007). Cette démarche a permis d'identifier 13 outils psychométriques (Tableau 1). Bien que les objectifs de certaines mesures se chevauchent, elles sont présentées en trois catégories: (1) les mesures ciblant les événements traumatiques; (2) les mesures de symptômes liés au traumatisme (par exemple: état de stress post-traumatique, dissociation), incluant aussi les mesures comportementales couramment utilisées dans le domaine des traumatismes; (3) les mesures qui incluent à la fois les événements traumatiques et les symptômes liés au traumatisme. Cette division est d'ailleurs cohérente avec la conceptualisation des troubles psychiatriques liés à des traumatismes (par ex.: État de stress post-traumatique, dans le DSM-V) où l'exposition à des événements traumatiques et la présentation de séquelles associées à ces expériences sont des volets essentiels au diagnostic. Les mesures spécifiques aux capacités d'adaptation et de résilience des jeunes ne sont pas incluses dans cette sélection, mais sont résumées en détail dans d'autres ouvrages (par exemple, Nader, 2008).

À quelques exceptions près, les questionnaires discutés dans la présente revue sont remplis par les jeunes. En effet, les jeunes sont considérés comme une source importante d'informations sur leurs réactions post-traumatiques et déclarent généralement plus de symptômes que des tiers (Nader, 2008). De nombreux chercheurs recommandent de favoriser les évaluations auto-rapportées pour mesurer les symptômes intériorisés, notamment les symptômes traumatiques, et d'utiliser des instruments complétés par les parents ou les enseignants pour corroborer des informations concernant les symptômes externalisés et les problèmes scolaires si nécessaire (Grills \& Ollendick, 2002; Weis et al., 2005 ; Nader, 2008). Ces outils peuvent servir à évaluer de façon préliminaire la situation globale d'un jeune et ainsi à identifier des domaines plus problématiques qui pourraient nécessiter une référence pour une évaluation spécialisée (Nader, 2008). En ce sens, l'utilisation de ces outils ne devrait en aucun cas remplacer une évaluation psychologique ou psychiatrique par un professionnel accrédité qui pourra établir des diagnostics plus précis.

Afin d'identifier les outils les plus pertinents pour une clientèle dans un contexte donné, il est essentiel d'examiner un certain nombre de questions pratiques additionnelles, notamment l'âge de la population d'intérêt, le nombre/type de questions, le temps d'administration, le 
TABLEAU 1

Revue des outils pertinents pour mesurer le trauma chez des jeunes en CR Événements traumatiques

\begin{tabular}{|c|c|c|}
\hline Questionnaires & Description & $\begin{array}{l}\text { Références pour } \\
\text { les qualités } \\
\text { psychométriques }\end{array}$ \\
\hline $\begin{array}{l}\text { Childhood Trauma } \\
\text { Questionnaire- } \\
\text { short form } \\
\text { (CTQ-SF) } \\
\text { (Bernstein, 1998) }\end{array}$ & $\begin{array}{l}\text { Type de mesure: Autorapportée } \\
\text { Nombre d'items: } 28 \\
\text { Clientèle visée: Adolescents de } 12 \text { ans } \\
\text { et plus } \\
\text { Sous-échelles: Mauvais traitements, } \\
\text { violence psychologique, négligence } \\
\text { physique, négligence émotionnelle, } \\
\text { agression sexuelle } \\
\text { Catégorie de réponses: Cinq points allant } \\
\text { de jamais vrai à très souvent vrai } \\
\text { Échelle de temps: Expériences passées } \\
\text { Échelle de minimisation et de déni: Oui } \\
\text { Temps alloué: } 5 \text { minutes } \\
\text { Distributeur: Pearson } \\
\text { (www.pearsonassessment.com) } \\
\text { Frais: Oui } \\
\text { Qualifications: Maîtrise ou équivalent }\end{array}$ & $\begin{array}{l}\text { Bernstein et al., } \\
\text { 1994, 1997, 2003; } \\
\text { Bernstein, 1998; } \\
\text { Hamby \& } \\
\text { Finkelhor, 2000; } \\
\text { Wright et al., } \\
\text { 2001; Scher et } \\
\text { al., 2001; Paivio } \\
\text { \& Cramer, 2004; } \\
\text { Margolin, 2005; } \\
\text { Garrusi \& } \\
\text { Nakhaee, 2009; } \\
\text { Thombs et al., } \\
\text { 2007; Forde } \\
\text { et al., 2012. }\end{array}$ \\
\hline $\begin{array}{l}\text { (1) Traumatic } \\
\text { Events Screening } \\
\text { Inventory-Child } \\
\text { (TESI-C; Ghosh } \\
\text { Ippen et al., } \\
\text { 2002) } \\
\text { (2) TESI-Self- } \\
\text { Report Revised } \\
\text { (TESI-SRR; Ford } \\
\text { et al., 2005) }\end{array}$ & $\begin{array}{l}\text { Type de mesure: (1) Entrevue structurée } \\
\text { et (2) Autorapportée } \\
\text { Nombre d'items: (1) } 6 \text { items et des } \\
\text { questions de suivi pour les détails } \\
\text { concernant chaque expérience } \\
\text { traumatique; (2) } 24 \text { items } \\
\text { Clientèle visée: De } 8 \text { à } 18 \text { ans } \\
\text { Sous-échelles: Blessures, hospitalisations, } \\
\text { violence dans la famille, violence dans la } \\
\text { communauté, désastres naturels, accidents, } \\
\text { agression physique, agression sexuelle } \\
\text { Catégorie de réponses: (1) } 4 \text { choix, soit } \\
\text { oui, non, incertain, refuse de répondre; } \\
\text { (2) Oui, non, refuse de répondre } \\
\text { Échelle de temps: Expériences passées et } \\
\text { 12 derniers mois } \\
\text { Échelle de minimisation et de déni: Non } \\
\text { Temps alloué: } 30 \text { minutes } \\
\text { Distributeur: Directement accessible en } \\
\text { ligne www.ptsd.va.gov/professional/ } \\
\text { pages/assessments/assessment-pdf/ } \\
\text { TESI-C.pdf, une permission d'utilisation } \\
\text { doit être adressée à ncptsd@ncptsd.org } \\
\text { Frais: Non } \\
\text { Qualifications: Professionnels dans le } \\
\text { domaine de la santé mentale }\end{array}$ & $\begin{array}{l}\text { Ribbe, 1996; } \\
\text { Ghosh Ippen et } \\
\text { al., 2002; Ford } \\
\text { et al., 2005. }\end{array}$ \\
\hline
\end{tabular}




\begin{tabular}{|c|c|c|}
\hline Questionnaires & Description & $\begin{array}{l}\text { Références pour } \\
\text { les qualités } \\
\text { psychométriques }\end{array}$ \\
\hline $\begin{array}{l}\text { Juvenile } \\
\text { Victimization } \\
\text { Questionnaire } \\
\text { - Youth self report } \\
\text { (JVQ-SR) (Hamby } \\
\text { et al., 2004) }\end{array}$ & $\begin{array}{l}\text { Type de mesure: Entrevue structurée } \\
\text { Nombre d'items: } 50 \text { et des questions de } \\
\text { suivi pour les détails concernant chaque } \\
\text { expérience de victimisation; une version } \\
\text { abrégée de } 34 \text { items a également été } \\
\text { développée } \\
\text { Clientèle visée: De } 8 \text { à } 17 \text { ans } \\
\text { Sous-échelles: Crime conventionnel, } \\
\text { mauvais traitements, victimisation dans la } \\
\text { fratrie et avec les pairs, victimisation } \\
\text { sexuelle, exposition indirecte et témoin } \\
\text { de violence } \\
\text { Catégorie de réponses: } 2 \text { choix, soit oui, } \\
\text { non } \\
\text { Échelle de temps: Expériences passées et } \\
12 \text { derniers mois } \\
\text { Échelle de minimisation et de déni: Non } \\
\text { Temps alloué: } 20-30 \text { minutes } \\
\text { Distributeur: Directement accessible en } \\
\text { ligne www.unh.edu/ccrc/pdf/jvq/JVQ_ } \\
\text { Self-reportScreeners_6_8_05.pdf, mais } \\
\text { une permission d'utilisation doit être } \\
\text { adressée à kelly.foster@unh.edu } \\
\text { Frais: Non } \\
\text { Qualifications: Non spécifié }\end{array}$ & $\begin{array}{l}\text { Hamby et al., } \\
2004 \text {; Finkelhor } \\
\text { et al., } 2005 .\end{array}$ \\
\hline $\begin{array}{l}\text { Dimensions of } \\
\text { Stressful Events } \\
\text { Rating Scale } \\
\text { (DOSE) (Fletcher, } \\
\text { 1996a) }\end{array}$ & $\begin{array}{l}\text { Type de mesure: Entrevue structurée } \\
\text { Nombre d'items: } 50 \text {, dont } 24 \text { spécifiques } \\
\text { aux agressions sexuelles } \\
\text { Clientèle visée: De } 0 \text { à } 17 \text { ans (sur la base } \\
\text { de réponses données par le parent si } \\
\text { nécessaire) } \\
\text { Sous-échelles: Fréquence des événements } \\
\text { traumatiques, sévérité, relation avec } \\
\text { l'agresseur sexuel, sentiment de stigmati- } \\
\text { sation, conflits moraux et religieux } \\
\text { Catégorie de réponses: } 2 \text { choix, soit oui, } \\
\text { non; ou échelle de fréquence } \\
\text { Échelle de temps: Expériences passées } \\
\text { Échelle de minimisation et de déni: Non } \\
\text { Temps alloué: Non spécifié } \\
\text { Distributeur: Accessible en contactant } \\
\text { l'auteur: } \\
\text { Kenneth.Fletcher@umassmed.edu } \\
\text { Frais: Non } \\
\text { Qualifications: Professionnels dans le } \\
\text { domaine de la santé mentale }\end{array}$ & $\begin{array}{l}\text { Fletcher (1996a, } \\
\text { 1996b, 2007); } \\
\text { Fletcher et al., } \\
\text { 2006; Spilsbury } \\
\text { et al., 2008. }\end{array}$ \\
\hline
\end{tabular}


TABLEAU 1 (suite)

Symptômes traumatiques

\begin{tabular}{|c|c|c|}
\hline Questionnaires & Description & $\begin{array}{l}\text { Références pour } \\
\text { les qualités } \\
\text { psychométriques }\end{array}$ \\
\hline $\begin{array}{l}\text { Trauma Symptom } \\
\text { Checklist for } \\
\text { Children (TSCC) } \\
\text { (Briere, 1996) }\end{array}$ & $\begin{array}{l}\text { Type de mesure: Autorapportée } \\
\text { Nombre d'items: } 54 \\
\text { Clientèle visée: De } 8 \text { à } 17 \text { ans } \\
\text { Sous-échelles: Anxiété, dépression, } \\
\text { colère, stress post-traumatique, } \\
\text { dissociation et préoccupations sexuelles } \\
\text { Catégorie de réponses: Quatre choix } \\
\text { allant de jamais à presque tout le temps } \\
\text { Échelle de temps: Non spécifié } \\
\text { Échelle de minimisation et de déni: 0ui } \\
\text { Temps alloué: } 20-30 \text { minutes } \\
\text { Distributeur: Psychological Assessment } \\
\text { Resources (PAR) www4.parinc.com/ } \\
\text { Products/Product.aspx? ProductID=TSCC } \\
\text { Frais: Oui } \\
\text { Qualifications: Clinicien formé avec un } \\
\text { diplôme de premier cycle }\end{array}$ & $\begin{array}{l}\text { Singer et al., } \\
\text { 1995; Briere, } \\
\text { 1996; Lanktree } \\
\text { et al., } 2008 .\end{array}$ \\
\hline $\begin{array}{l}\text { Adolescent } \\
\text { Dissociative } \\
\text { Experience Scale } \\
\text { (A-DES) } \\
\text { (Armstrong } \\
\text { et al., 1997) }\end{array}$ & $\begin{array}{l}\text { Type de mesure: Entrevue structurée } \\
\text { Nombre d'items: } 30 \\
\text { Clientèle visée: De } 11 \text { à } 16 \text { ans } \\
\text { Sous-échelles: Amnésie dissociative, } \\
\text { absorption et recours à l'imaginaire } \\
\text { imaginative, influence passive, } \\
\text { dépersonnalisation et déréalisation } \\
\text { Catégorie de réponses: Onze choix allant } \\
\text { de jamais à toujours (0 à 10) } \\
\text { Échelle de temps: Non spécifié } \\
\text { Échelle de minimisation et de déni: Non } \\
\text { Temps alloué: } 25 \text { minutes } \\
\text { Distributeur: Accessible en contactant } \\
\text { l'auteur: jarmstrong@mizar.usc.edu } \\
\text { Frais: Minimaux - frais postaux } \\
\text { Qualifications: Clinicien formé avec un } \\
\text { diplôme de premier cycle }\end{array}$ & $\begin{array}{l}\text { Smith \& Carlson, } \\
1996 ; \text { Armstrong } \\
\text { et al., 1997; } \\
\text { Farrington et al., } \\
\text { 2001; Muris } \\
\text { et al., 2003. }\end{array}$ \\
\hline
\end{tabular}




\begin{tabular}{|c|c|c|}
\hline Questionnaires & Description & $\begin{array}{l}\text { Références pour } \\
\text { les qualités } \\
\text { psychométriques }\end{array}$ \\
\hline $\begin{array}{l}\text { Clinician- } \\
\text { Administered PTSD } \\
\text { Scale for Children } \\
\text { and Adolescents } \\
\text { (CAPS-CA) (Nader } \\
\text { et al., 1996) }\end{array}$ & $\begin{array}{l}\text { Type de mesure: Entrevue structurée } \\
\text { Nombre d'items: } 33 \\
\text { Clientèle visée: De } 8 \text { à } 18 \text { ans } \\
\text { Sous-échelles: Détresse globale, } \\
\text { capacités d'adaptation et déficiences } \\
\text { Catégorie de réponse: Cinq choix allant } \\
\text { de jamais à toujours } \\
\text { Échelle de temps: Les } 30 \text { derniers jours } \\
\text { Échelle de minimisation et de déni: Non } \\
\text { Temps alloué: } 30-60 \text { minutes } \\
\text { Distributeur: National Center for PTSD } \\
\text { www.ptsd.va.gov/professional/pages/ } \\
\text { assessments/caps-ca.asp } \\
\text { Frais: Non } \\
\text { Qualifications: Professionnels de la santé } \\
\text { mentale qualifiés et chercheurs; une } \\
\text { formation est nécessaire, mais des outils } \\
\text { d'autoapprentissage sont disponibles } \\
\text { gratuitement }\end{array}$ & $\begin{array}{l}\text { Hovens et al., } \\
\text { 1994; Blake et.al., } \\
\text { 1995; Mueser } \\
\text { et.al, 2000; } \\
\text { Weathers et al., } \\
\text { 2001. }\end{array}$ \\
\hline $\begin{array}{l}\text { Behavior } \\
\text { Assessment System } \\
\text { for Children-Self- } \\
\text { Report of } \\
\text { Personality } \\
\text { (BASC-2 SRP-A) } \\
\text { (Reynolds \& } \\
\text { Kamphaus, 2004) }\end{array}$ & $\begin{array}{l}\text { Type de mesure: Autorapportée } \\
\text { Nombre d'items: } 176 \\
\text { Clientèle visée: De } 12 \text { à } 21 \text { ans } \\
\text { Sous-échelles: Problèmes internalisés et } \\
\text { externalisés, problèmes scolaires, } \\
\text { fonctionnement adaptatif } \\
\text { Catégorie de réponses: Des questions de } \\
\text { type vrai ou faux / quatre choix, allant de } \\
\text { jamais à presque toujours } \\
\text { Échelle de temps: Temps présent } \\
\text { Échelle de minimisation et de déni : Oui, } \\
\text { trois indices de validité } \\
\text { Temps alloué: } 30 \text { minutes } \\
\text { Distributeur: Pearson http://www. } \\
\text { pearsonassessments.com/HAIWEB/ } \\
\text { Cultures/en-us/Productdetail.htm? } \\
\text { Pid=PAa30000 } \\
\text { Frais: Oui } \\
\text { Qualifications: Non spécifié; deux } \\
\text { logiciels sont disponibles pour aider à } \\
\text { simplifier le processus de notation et de } \\
\text { reportage, de même que des vidéos de } \\
\text { formation }\end{array}$ & $\begin{array}{l}\text { Doyle et al., } \\
\text { 1997; Reynolds \& } \\
\text { Kamphaus, 2004; } \\
\text { Tan, 2007; Weis \& } \\
\text { Smenner, 2007; } \\
\text { Frick et al., 2009. }\end{array}$ \\
\hline
\end{tabular}


TABLEAU 1 (suite)

\section{Symptômes traumatiques}

\begin{tabular}{|c|c|c|}
\hline Questionnaires & Description & $\begin{array}{l}\text { Références pour } \\
\text { les qualités } \\
\text { psychométriques }\end{array}$ \\
\hline $\begin{array}{l}\text { Child Behavior } \\
\text { Checklist for } \\
\text { Children (CBCL) } \\
\text { and YSR (Youth } \\
\text { Self-Report) } \\
\text { (Achenbach, 1991) }\end{array}$ & $\begin{array}{l}\text { Type de mesure: Autorapportée ou rapportée } \\
\text { par un adulte significatif pour l'enfant } \\
\text { Nombre d'items: } 112 \\
\text { Clientèle visée: De } 11 \text { à } 18 \text { ans } \\
\text { Sous échelles: Anxieux/déprimé, retiré/ } \\
\text { déprimé, plaintes somatiques, problèmes } \\
\text { sociaux, problèmes de la pensée, } \\
\text { problèmes d'attention, comportements } \\
\text { déviants, comportements agressifs } \\
\text { Catégorie de réponses: Trois choix, soit } \\
\text { pas vrai, souvent vrai ou très vrai } \\
\text { Échelle de temps: Les } 6 \text { derniers mois } \\
\text { Échelle de minimisation et de déni: Non } \\
\text { Temps alloué: } 10 \text { minutes } \\
\text { Distributeur: ASEBA } \\
\text { www.aseba.org/ordering/howtoorder.html } \\
\text { Frais: Oui } \\
\text { Qualifications: Maîtrise ou équivalent }\end{array}$ & $\begin{array}{l}\text { Ivanovna et al., } \\
\text { 2007; Warnick et } \\
\text { al., } 2007 \text {. }\end{array}$ \\
\hline
\end{tabular}

Événements et symptômes traumatiques

\begin{tabular}{|c|c|c|}
\hline Questionnaires & Description & $\begin{array}{l}\text { Références pour } \\
\text { les qualités } \\
\text { psychométriques }\end{array}$ \\
\hline $\begin{array}{l}\text { UCLA PTSD } \\
\text { Reaction Index for } \\
\text { DSM-IV (UPID) } \\
\text { (Pynoos et al., } \\
1998 \text { ) }\end{array}$ & $\begin{array}{l}\text { Type de mesure: Autorapportée } \\
\text { Nombre d'items: } 48 \\
\text { Clientèle visée: De } 7 \text { à } 18 \text { ans } \\
\text { Sous-échelles: Exposition à } 26 \text { événe- } \\
\text { ments traumatiques et questions } \\
\text { additionnelles pour évaluer le sentiment } \\
\text { de honte, l'agressivité et la dissociation } \\
\text { Catégorie de réponses: Oui/non (événe- } \\
\text { ments traumatiques)/cinq choix allant de } \\
\text { jamais à la plupart de temps (symptômes) } \\
\text { Échelle de temps: Expériences passées } \\
\text { (événements traumatiques)/ Les } 30 \\
\text { derniers jours (symptômes vécus) } \\
\text { Échelle de minimisation et de déni: Non } \\
\text { Temps alloué: } 20-30 \text { minutes } \\
\text { Distributeur: UCLA Trauma Psychiatry } \\
\text { Service, } 300 \text { Medical Plaza, Los Angeles, } \\
\text { CA } 90095-6968 \\
\text { Téléphone: } 1310 \text { 206-8973, } \\
\text { HFinley@mednet.ucla.edu } \\
\text { Frais: Non } \\
\text { Qualifications: Professionnels dans le } \\
\text { domaine de la santé mentale }\end{array}$ & $\begin{array}{l}\text { Rodriguez et al. } \\
2001 ; \text { Roussos } \\
\text { et al., } 2005 .\end{array}$ \\
\hline
\end{tabular}




\begin{tabular}{|c|c|c|}
\hline Questionnaires & Description & $\begin{array}{l}\text { Références pour } \\
\text { les qualités } \\
\text { psychométriques }\end{array}$ \\
\hline $\begin{array}{l}\text { Child and } \\
\text { Adolescent Needs } \\
\text { and Strengths } \\
\text { (CANS) (Lyons et } \\
\text { al., 1999; 2008) }\end{array}$ & $\begin{array}{l}\text { Type de mesure: Grille clinique } \\
\text { Nombre d'items: } 100 \\
\text { Clientèle visée: De } 0 \text { à } 18 \text { ans } \\
\text { Sous-échelles: Expériences traumatiques, } \\
\text { symptômes traumatiques, forces de } \\
\text { l'enfant, domaines de fonctionnement, } \\
\text { acculturation, besoins émotifs et } \\
\text { comportementaux de l'enfant, comporte- } \\
\text { ments à risque de l'enfant, besoins vécus } \\
\text { dans la petite enfance, besoins liés au } \\
\text { logement autonome, besoins et forces des } \\
\text { parents ou substituts } \\
\text { Catégorie de réponses: Variable } \\
\text { Échelle de temps: Expériences passées/ } \\
\text { Symptômes vécus dans le dernier mois } \\
\text { Échelle de minimisation et de déni: Non } \\
\text { Temps alloué: Variable } \\
\text { Distributeur: Praed Foundation: } \\
\text { www.praedfoundation.org. Aussi } \\
\text { accessible en contactant l'auteur: } \\
\text { jlyons@uottawa.ca } \\
\text { Frais: Non } \\
\text { Qualifications: Clinicien formé avec un } \\
\text { diplôme de premier cycle; expertise en } \\
\text { santé mentale requise pour les sections } \\
\text { portant sur les troubles mentaux }\end{array}$ & $\begin{array}{l}\text { Lyons et al., } \\
2000 ; \text { Anderson } \\
\text { et al., 2003; } \\
\text { Lyons, 2004; } \\
\text { Dilley et al., } \\
\text { 2007; Sieracki } \\
\text { et al., } 2008\end{array}$ \\
\hline $\begin{array}{l}\text { Child Welfare } \\
\text { Trauma Referral } \\
\text { Tool (CWT) (Taylor } \\
\text { et al., 2006) }\end{array}$ & $\begin{array}{l}\text { Type de mesure: Grille clinique } \\
\text { Nombre d'items: } 100 \\
\text { Clientèle visée: De } 0 \text { à } 18 \text { ans } \\
\text { Sous échelles: } 6 \text { sections: Expériences } \\
\text { traumatiques, symptômes traumatiques } \\
\text { actuels, problèmes d'attachement, } \\
\text { comportements nécessitant une } \\
\text { stabilisation immédiate, domaines de } \\
\text { fonctionnement actuel, arbre décisionnel } \\
\text { Catégorie de réponses: Oui, non, } \\
\text { soupçonné, inconnu } \\
\text { Échelle de temps: Expériences passées/ } \\
\text { symptômes vécus actuellement (non } \\
\text { spécifié) } \\
\text { Échelle de minimisation et de déni: Non } \\
\text { Temps alloué: Variable } \\
\text { Distributeur: Directement accessible en } \\
\text { ligne } \\
\text { www.nctsnet.org/nctsn_assets/pdfs/ } \\
\text { CWT3_SH0_Referral.pdf } \\
\text { Frais: Aucun } \\
\text { Qualifications: Clinicien formé avec un } \\
\text { diplôme de premier cycle }\end{array}$ & $\begin{array}{l}\text { Taylor et al., } \\
2006\end{array}$ \\
\hline
\end{tabular}


coût, la disponibilité de l'instrument dans diverses langues, la convivialité de la notation, les qualités psychométriques, les qualifications nécessaires pour l'administration de l'outil, et la faisabilité globale de l'implantation de l'outil dans un milieu de pratique (Ohan et al., 2002; Strand et al., 2005 ; Nader, 2008). Le tableau 1 offre un certain nombre d'informations à ce sujet pour chacun des outils présentés. En ce qui concerne l'âge de la population d'intérêt, Amaya-Jackson et ses collègues (2000), ainsi que Hamby et Finkelhor (2000), recommandent de choisir des outils à la lumière de l'âge développemental de l'enfant et non uniquement en prenant en compte son âge chronologique, et de considérer cette information dans l'interprétation des résultats. En outre, la sélection devra être basée sur une réflexion quant à l'objet d'intérêt, c'est-à-dire les événements traumatiques, les symptômes traumatiques ou ces deux construits simultanément. Afin de guider ce choix, quelques considérations sont discutées ici au sujet de chacune des options.

\section{Évaluation des événements traumatiques}

Finkelhor et ses collègues (2007) préconisent de questionner les jeunes au sujet des événements traumatiques qu'ils pourraient avoir vécus, compte tenu de leur impact important sur le fonctionnement à travers différents domaines. Cela est particulièrement vrai pour les enfants qui ont vécu de multiples formes de traumatismes et qui seraient encore plus à risque de présenter des séquelles. Afin de promouvoir le bien-être des enfants et des jeunes à long terme, il ne suffit pas de se concentrer uniquement sur leurs réactions psychologiques ou leurs symptômes; il faut également mieux cerner la nature des événements traumatiques afin d'identifier les facteurs contextuels qui peuvent augmenter le risque de trauma futur et élever le risque de séquelles à long terme (Stevenson, 1999). Une mesure des événements vécus devrait idéalement couvrir à la fois les expériences de la dernière année et également celles vécues à vie, afin de comprendre la trajectoire de ces jeunes et les moments clés dans leur développement où les traumatismes ont été vécus. Puisque les événements traumatiques varient en nature, intensité et durée, une information détaillée des expériences est aussi souhaitée puisqu'elle aide les cliniciens à comprendre les réactions des jeunes, à mieux identifier les rappels traumatiques (déclencheurs), et à prévoir les modes de comportement (Nader, 2008). Une mesure directe des événements traumatiques peut également donner l'occasion aux jeunes de partager 
leurs expériences, dans certains cas, pour la première fois. Ce type de mesure peut donner l'opportunité à un enfant de briser le silence au sujet d'événements traumatiques qui, autrement, n'auraient pas été dévoilés.

Quatre questionnaires sont proposés pour mesurer les événements traumatiques (voir tableau 1). Le Childhood Trauma Questionnaire - Short Form (Bernstein, 1998) évalue chez les jeunes de 12 ans et plus cinq formes de mauvais traitements sur une échelle de fréquence: la violence physique ou psychologique, la négligence physique ou psychologique, et l'agression sexuelle. Le Traumatic Events Screening Inventory - Child Report et Self-Report Revised (Ghosh-Ippen et al., 2002; Ford et al., 2005) est une entrevue structurée menée auprès de jeunes de huit ans et plus qui évalue la présence d'événements traumatiques dans la famille et la communauté, incluant des catastrophes naturelles et des accidents. Dans cette même lignée, le Juvenile Victimization Questionnaire (Hamby et al., 2004) est une entrevue structurée qui documente les expériences de traumatismes à travers différents domaines, notamment des formes indirectes de victimisation (par exemple: être témoin de violence conjugale). Finalement, la Dimensions of Stressful Events Rating Scale (Fletcher, 1996a) se distingue des trois autres par sa mesure du degré d'impact perçu par les jeunes au sujet des expériences traumatiques vécues.

Lutilisation de ces outils doit s'accompagner d'une réflexion éthique. En effet, Becker-Blease \& Freyd (2006) et Cashmore (2006) soulignent les enjeux importants que ces évaluations comportent, notamment ceux liés à la confidentialité et au risque de stigmatisation. En lien avec la confidentialité, il est important de mentionner que la législation varie d'un pays à l'autre concernant l'obligation de signaler aux autorités des révélations de mauvais traitements et de négligence. Alors que de nombreux pays ont mis en place une telle structure légale, d'autres n'ont pas cette obligation, notamment l'Angleterre, l'Allemagne et la NouvelleZélande (Matthews \& Kenny, 2008). Ainsi, les jeunes qui remplissent ces questionnaires devraient obtenir au préalable une information éclairée afin de connaître les procédures qui pourraient être mises en place dans l'éventualité d'un dévoilement. En outre, afin de diminuer les risques de stigmatisation pouvant être vécue après la passation d'un questionnaire, Cashmore (2006) recommande d'implanter une procédure formelle de suivi afin de détecter toute forme d'inconfort et de malaise, voire de détresse, induit par la révélation de situations traumatisantes. 


\section{Évaluation des symptômes liés aux traumatismes}

Selon Brière et Lanktree (2008), une évaluation optimale des enfants et des jeunes doit inclure une description du fonctionnement psychologique actuel et des cibles potentielles de traitement. Étant donné la nature complexe des traumatismes, ces auteurs recommandent l'administration de plusieurs mesures qui puisent dans une variété de symptômes. À cet effet, des mesures unidimensionnelles ou multidimensionnelles peuvent constituer cette batterie. Les mesures unidimensionnelles fournissent plus d'informations sur une seule dimension et génèrent un portrait sommaire de ce problème en particulier. Les mesures unidimensionnelles les plus communes dans le domaine du trauma sont celles mesurant l'état de stress post-traumatique et la dissociation. Les mesures de l'état de stress post-traumatique portent sur la formulation de ce trouble dans le DSM-IV (American Psychological Association, 2000), c'est-à-dire les symptômes d'évitement, d'intrusion et d'hyperéveil. De la même façon, les mesures de dissociation incluent, par exemple, des expériences de dépersonnalisation, d'amnésie et d'absorption. De façon générale, des normes sont établies pour ces échelles et permettent d'établir si un jeune présente des symptômes jugés cliniques. Les mesures retenues dans cette revue sont la Clinican Administered PTDS Scale for Children and Adolescents (Nader et al., 1996) pour évaluer l'état de stress post-traumatique et l'Adolescent Dissociative Experience Scale (Armstrong et al., 1997) pour identifier les symptômes de dissociation. La première est une entrevue semi-structurée visant les enfants de huit ans et plus qui permet d'établir une impression diagnostique d'état de stress post-traumatique, alors que la seconde est un questionnaire auto-rapporté qui évalue les formes normatives et pathologiques de dissociation.

Les mesures multidimensionnelles sont plus globales et permettent de synthétiser l'information sur de nombreux aspects et de les condenser en différents indices cliniques. Ces mesures incluent des items se rapportant à différents construits, dont le stress post-traumatique et la dissociation, mais aussi l'anxiété, la dépression, la colère, les préoccupations sexuelles, la perception négative de soi, les troubles alimentaires, l'automutilation, la culpabilité et la honte, dont certains sont considérés comme des symptômes d'un traumatisme complexe (van der Kolk \& Courtois, 2005). L'instrument le plus reconnu pour documenter ce construit multidimensionnel est la Trauma Symptom Checklist for Children qui permet d'évaluer les symptômes de colère, de dépression, d'an- 
xiété, de stress post-traumatique, de dissociation et de préoccupations sexuelles chez les enfants et jeunes de 8 à 16 ans (Briere, 1996). Certaines mesures peuvent également viser à documenter les problèmes de comportement, qui sont extrêmement fréquents chez les enfants et les jeunes traumatisés (Briere \& Lanktree, 2008; NCTSN, 2008), et vus comme un mécanisme de réaction au trauma omniprésent chez les adolescents en particulier (Brière, 1996; Archer, 2005). Parmi les outils les plus utilisés auprès d'une clientèle traumatisée se retrouvent le Youth Self-Report (Achenbach, 1991), le Behavior Assessment System for Children-Self-Report of Personality (Reynolds \& Kamphaus, 2004), et le Strength and Difficulties Questionnaire (SDQ) (Goodman, 1997). Le premier constitue un incontournable dans le domaine de l'évaluation de symptômes internalisés et externalisés chez les adolescents, symptômes qui se regroupent autour de huit échelles: retrait social, plaintes somatiques, dépression/anxiété, difficultés sociales, troubles de la pensée, problèmes d'attention, comportements délinquants et attitudes agressives. Les deux autres outils sont également des mesures autorapportées, le premier se concentrant sur les symptômes internalisés et externalisés, mais également sur les problèmes scolaires et le fonctionnement adaptatif, et le second sur des symptômes similaires mais aussi sur la présence de comportements prosociaux. Pour ces trois questionnaires, une version équivalente à celle remplie par les jeunes est disponible pour les parents ou autres adultes significatifs pour l'enfant.

\section{Évaluation des événements et des symptômes traumatiques}

Peu d'instruments de mesure visent en même temps les événements traumatiques et leurs symptômes. Ces outils ont le mérite d'englober à la fois la source du trauma ainsi que ses séquelles, mais ne sont pas nécessairement aussi holistiques que les mesures multidimensionnelles présentées plus haut. Par exemple, le UCLA PTSD Reaction Index for $D S M-I V$ (Pynoos et al., 1998) est un questionnaire fort reconnu pour documenter, chez les enfants de 7 à 18 ans, les événements traumatiques et la constellation de symptômes de l'état de stress post-traumatique, mais n'inclut aucun item concernant les autres conséquences possibles du trauma. Le Child and Adolescent Needs and Strengths (Lyons et al., 1999, 2008) et le Child Welfare Trauma Referral Tool (Taylor et al., 2006) sont deux outils cliniques utilisés par les intervenants sur 
la base d'information provenant de diverses évaluations, du dossier et de discussions cliniques. Ces instruments n'offrent pas de normes et ne sont pas utilisés pour évaluer la gravité ou la fréquence de symptômes. Plutôt, ils permettent aux intervenants d'organiser l'information au sujet d'un jeune de façon cohésive et ciblée, à la fois tant sur les événements traumatiques que les séquelles, ce qui peut améliorer les discussions en équipe et la prise de décision pour le suivi. Le Child Welfare Trauma Referral Tool comporte également un arbre décisionnel qui permet, à la lumière des informations recueillies, de prendre une décision quant au type de traitement le plus approprié en regard de la situation actuelle. Par exemple, ce modèle préconise, pour un jeune traumatisé et aux prises avec des problèmes importants de toxicomanie, d'entreprendre d'abord une démarche pour réduire la consommation de substances et ensuite d'intégrer une intervention spécifique au trauma.

\section{Conclusion}

Le présent article visait à offrir une synthèse des outils psychométriques pour évaluer le trauma chez les jeunes en CR, dans le but de rendre plus accessible cette information nécessaire à la transformation des services traditionnels de protection de l'enfance en un programme solide et cohésif de santé mentale axé sur le traumatisme. Les outils révisés incluent des mesures qui doivent être choisies selon un certain nombre de considérations pratiques, notamment l'âge de la clientèle visée, la formation nécessaire pour la passation du questionnaire, le temps de passation, mais qui doivent aussi être sélectionnés selon l'objectif d'évaluation poursuivi, soit de mieux comprendre les événements traumatiques, les symptômes traumatiques ou ces deux construits simultanément. Considérant le peu de mesures portant à la fois sur les événements et les séquelles traumatiques, il s'avère pertinent de recommander l'utilisation de mesures multiples et complémentaires afin d'identifier les jeunes à risque, de référer à une évaluation plus spécialisée au besoin, et d'orienter les services (Nader, 2008). Aussi, la passation de ces questionnaires doit s'accompagner d'une réflexion quant aux enjeux de confidentialité lors du dévoilement de traumatismes inconnus jusqu'alors des services de la protection de la jeunesse, et au potentiel de stigmatisation et de détresse induit lors de la divulgation des événements traumatiques. 
En outre, il est important de considérer que les symptômes liés aux traumatismes et ceux à des problèmes de santé mentale peuvent se confondre et se chevaucher, ce qui complique leur évaluation respective (American Academy of Child and Adolescent Psychiatry, 2002). Par exemple, l'évitement et l'hyperéveil peuvent être des symptômes d'exposition à un traumatisme ou le reflet d'un autre trouble anxieux, tout comme le retrait social, les problèmes de sommeil et une perte d'intérêt peuvent être des symptômes d'exposition à un traumatisme ou l'indication d'un trouble de dépression majeure. En outre, un enfant peut avoir un problème de santé mentale sans avoir vécu un événement traumatisant, comme un enfant peut avoir vécu un événement traumatisant sans souffrir d'un trouble. Ce manque de distinction est compliqué par le fait qu'un événement traumatique peut aggraver un trouble mental déjà présent ou pour lequel l'enfant cumulait un certain nombre de facteurs de risque génétiques et environnementaux avant même l'exposition aux traumas, indiquant qu'un enfant peut être à la fois traumatisé et affecté d'un trouble mental. Le choix d'outils psychométriques, de même que l'analyse de leurs résultats, doivent donc s'effectuer sur la base d'une démarche rigoureuse qui prend en compte une multitude d'informations et un jugement clinique éclairé (Frick et al., 2009). Outre ces considérations, il est important de rappeler qu'il n'est pas avisé d'établir des prédictions à long terme sur la base des résultats aux questionnaires, considérant que l'adolescence constitue une période de changements importants. Les résultats aux échelles de troubles mentaux et comportementaux doivent ainsi être vus comme une mesure de l'état actuel de la situation, et non une prédiction absolue des enjeux futurs que ces jeunes pourraient présenter lors de la transition à la vie adulte.

L'implantation d'une évaluation systématique des traumas pour les enfants et jeunes en CR pourrait servir, optimalement, de tremplin vers le développement de services spécialisés pour cette clientèle à risque. À cet effet, il est fort intéressant de voir se déployer des recherches évaluatives qui visent à démontrer l'efficacité de traitements basés sur l'évidence pour les jeunes en CR. Récemment, Weiner et ses collègues (2009) ont démontré l'efficacité de trois programmes de traitement reconnus, la Child-Parent Psychotherapy, la Trauma-Focused Cognitive Behavioral Therapy, et la Stuctured Psychotherapy for Adolescents Responding to Chronic Stress, auprès d'une clientèle adolescente en situation de placement. Malgré les changements apportés aux programmes initiaux 
pour adapter les interventions à la situation de placement des jeunes, les 216 participants à l'une ou l'autre de ces interventions ont présenté une réduction de leurs symptômes et une augmentation de leur niveau de fonctionnement après avoir pris part à la thérapie. Toutefois, ces jeunes n'étaient pas comparés à ceux recevant des services usuels et, en ce sens, l'étude ne peut conclure que ces programmes sont plus efficaces que les services habituels. L'application de TF-CBT auprès d'enfants et d'adolescents ayant vécu des traumas multiples a été discutée récemment par les auteurs de cette approche (voir par exemple Cohen et al., 2012), ce qui semble une avenue fort encourageante quoique son efficacité auprès de jeunes en CR devra être établie dans des recherches futures.

D'autres interventions sont prometteuses, notamment le modèle Attachment, Regulation, and Competency (Kinniburgh et al., 2005 ; Blaustein \& Kinniburgh, 2010) qui a fait l'objet d'études préliminaires témoignant d'un potentiel pour son application auprès de populations en situation de placement (Arvidson et al., 2011), mais qui doit, aussi, être évalué de façon plus formelle pour des adolescents en CR. L'avantage de ce modèle d'intervention est qu'il vise non seulement à traiter directement les séquelles des enfants et des jeunes, mais aussi à outiller les intervenants qui travaillent sur le terrain au quotidien avec cette clientèle et à modifier les systèmes dans lesquels les jeunes gravitent pour les rendre plus susceptibles de favoriser des changements chez les enfants et les adolescents traumatisés. Ce modèle récemment publié par des chercheurs et cliniciens du NCTSN est fondé sur les théories du trauma, de l'attachement et du développement de l'enfant. Des techniques cognitivo-comportementales et psychoéducatives forment l'essentiel de ce modèle d'intervention, bien que celles-ci sont complétées, par exemple, par des techniques de relaxation et des approches créatives (art-thérapie). Ce modèle a été développé pour des enfants et adolescents qui ont vécu une diversité d'événements traumatiques et adverses et qui présentent des séquelles dans leur fonctionnement émotif, social et comportemental. Le modèle identifie trois principaux domaines que les intervenants doivent cibler pour aider les enfants et les adolescents à développer leurs forces et compétences, et réduire les facteurs de risque qui perpétuent les séquelles liées au trauma: l'attachement, la régulation des affects et le développement des compétences, trois grands domaines fortement altérés chez les enfants ayant vécu des traumatismes (D’Andrea et al., 2012). Ce type d'intervention pourrait répondre à un énoncé de l'Association des Centres jeunesse du Québec (ACJQ) qui recommandait d'offrir au 
personnel éducateur des CR un milieu professionnel plus soutenant, en ajoutant des ressources en supervision et en soutien (ACJQ, 2004). Considérant le manque de ressources financières que connaît le domaine de la protection de l'enfance, l'implantation d'interventions rentables, telles que celles misant sur les ressources existantes, dont le personnel œuvrant dans les unités de $\mathrm{CR}$, est une avenue à privilégier. Des études récentes suggèrent que l'accroissement des connaissances et le renforcement des compétences des intervenants en protection de la jeunesse, par l'entremise de formation et de supervision, peuvent être des moyens efficaces pour améliorer la santé mentale des jeunes auprès desquels ils interviennent, puisque les intervenants sont alors plus à même de mettre en place des interventions efficaces et basées sur des preuves empiriques (Cooley \& Petren, 2011; Glisson et al., 2011). Ces études démontrent également que les intervenants acquièrent un sentiment d'efficacité personnelle plus important et ont une perception plus positive de la qualité du milieu dans lequel ils travaillent, ce qui pourrait indirectement influencer le bien-être des jeunes en CR.

En bref, afin de répondre à l'éventail de besoins et de particularités des enfants et adolescents victimes de traumatismes et placés en CR, il est souhaitable que des avenues d'intervention soient explorées et validées afin que se bonifie le bassin existant de pratiques jugées exemplaires dans ce domaine. Cette démarche ne pourrait s'effectuer sans une réflexion quant au choix de mesures évaluatives pour documenter les événements traumatiques et les séquelles associées. Nous espérons que la présente revue contribuera à cette réflexion en offrant aux chercheurs et praticiens œuvrant auprès des jeunes en CR une source d'information quant aux options possibles et leurs considérations pratiques et méthodologiques.

\section{Références}

Achenbach, T. M. (1991). Manual for the Child Behavior Checklist/4-18 and 1991 Profile. Burlington, VT: University of Vermont Department of Psychiatry.

Amaya-Jackson, L., Socolar, R. R. S., Hunter, W., Runyan, D. K., et Colindres, R. (2000). Directly questioning children and adolescents about maltreatment: A review of survey measures used. Journal of Interpersonal Violence, 15 (7), 725-759.

American Academy of Child and Adolescent Psychiatry and Child Welfare League of America (2002). AACAP/CWLA Policy Statement on Mental Health 
and Substance Use Screening and Assessment of Children in Foster Care. American Academy of Child and Adolescent Psychiatry and Child Welfare League of America.

American Psychological Association (2000). Diagnostic and Statistical Manual of Mental Disorders (Revised, 4th ed.). Washington, DC: American Psychiatric Association.

Anderson, R. L., Lyons, J. S., Giles, D. M., Price, J. A., \& Estle, G. (2003). Reliability of the Child and Adolescent Needs and Strengths - Mental Health (CANS-MH) scale. Journal of Child and Family Studies, 12 (3), 279-289.

Archer, R. P. (2005). MMPI-A: Assessing Adolescent Psychopathology (3rd Ed.). New Jersey: Lawrence Erlbaum Associates, Inc.

Armstrong, J., Putnam, F., Carlson, E., Libero, D., \& Smith, S. (1997). Development and validation of a measure of adolescent dissociation: The Adolescent Dissociative Experiences Scale. Journal of Nervous and Mental Disorders, 185 (8), 491-497.

Arvidson, J., Kinniburgh, K., Howard, K., Spinazzola, J., Strothers, H., Evans, M., Andres, B., Cohen, C., \& Blaustein, M.E. (2011). Treatment of complex trauma in young children: Developmental and cultural considerations in application of the ARC intervention model. Journal of Child $\mathcal{\sigma}$ Adolescent Trauma, 4 (1), 34-51.

Association des Centres jeunesse du Québec (2004). Les centres jeunesse savent eux aussi que les besoins des jeunes sont grands. Communiqué de presse: http:// www.acjq.qc.ca/ ? 99E56A56-DDD7-41EB-9BBE-B79F22706603

Baker, A. J. L., Curtis, P. A., \& Papa-Lentini, C. (2006). Sexual abuse histories of youth in child welfare residential treatment centers: Analysis of the Odyssey Project Population. Journal of Child Sexual Abuse: Research, Treatment, $\mathcal{W}$ Program Innovations for Victims, Survivors, $\mathfrak{N}$ Offenders, 15 (1), 29-49.

Baker, A. J. L., Kurland, D., Curtis, P., Alexander, G., \& Papa-Lentini, C. (2007). Mental health and behavioral problems of youth in the child welfare system: Residential treatment centers compared to therapeutic foster care in the Odyssey Project population. Child Welfare: Journal of Policy, Practice, and Program, 86 (3), 97-123.

Baker, A. J. L., Piotrkowski, C. S, et Mincer, C. (2003). Behavioral predictors of psychiatric emergency in a child welfare residential treatment center. Residential Treatment for Children and Youth, 21 (1), 51-70.

Becker-Blease, K. A., et Freyd, J. J. (2006). Research participants telling the truth about their lives. American Psychologist, 61 (3), 218-226.

Bernstein, D. P. (1998). Childhood Trauma Questionnaire: A retrospective self-report. San Antonio, TX: The Psychological Corporation.

Bernstein, D. P., Ahluvalia, T., Pogge, D., \& Handelsman, L. (1997). Validity of the Childhood Trauma Questionnaire in an adolescent psychiatric population. Journal of the American Academy of Child and Adolescent Psychiatry, 36 (3), 340-348.

Bernstein, D. P., Fink, L. Handelsman, L., Foote, J., Lovejoy, M., Wenzel, K., Sapareto, E., \& Ruggiero, J. (1994). Initial reliability and validity of a new 
retrospective measure of child abuse and neglect. American Journal of Psychiatry, 151 (8), 1132-1136.

Bernstein, D. P., \& Fink, L. (1998). Childhood Trauma Questionnaire: A retrospective self-report. San Antonio, TX: The Psychological Corporation.

Bernstein, D. P., Stein, J. A., Newcomb, M. D., Walker, D., Pogge, D., Ahluvalia, T., Stokes, J., Handelsman, L., Medrano, M., Desmond, D., \& Zule, W. (2003). Development and validation of a brief screening version of the Childhood Trauma Questionnaire. Child Abuse o Neglect, 27 (2), 169-190.

Betterman, J. E., \& Jasperson, J. A. (2009). Adolescents in residential and inpatient treatment: A review of the outcome literature. Child and Youth Care Forum, 38 (4), 161-183.

Blake, D. D., Weathers, F. W., Nagy, L. M., Kaloupek, D. G., Gusman, F. D., Charney, D. S., \& Keane, T. M. (1995). The development of a clinicianadministered PTSD scale. Journal of Traumatic Stress, 8 (1), 75-90.

Blaustein, M. E., \& Kinninburgh, K. M. (2010). Treating Traumatic Stress in Children and Adolescents: How to Foster Resilience through Attachment, Self-Regulation, and Competency. New York, NY: Guildford Press.

Brady, L. K., \& Caraway, J. S. (2002). Home away from home: Factors associated with current functioning in children living in a residential treatment setting. Child Abuse and Neglect, 26 (11), 1149-1163.

Briere, J. (1996). Trauma symptom checklist for Children: Professional manual. Florida: Psychological Assessment Resources.

Briere, J., \& Lanktree, C. (2008). Integrative treatment of complex trauma for adolescents (ITCT-A): A guide for the treatment of multiply-traumatized youth. Retrieved from JohnBriere.com.

Burns, B. J., Phillips, S. D., Wagner, H. R., Barth, R. P., Kolko, D. J., Campbell, Y., \& Landsverk, J. (2004). Mental health need and access to mental health services by youths involved with child welfare: A national survey. Journal of the American Academy of Child $\mathcal{F}$ Adolescent Psychiatry, 43 (8), 960-970.

Carter, A. S., Briggs-Gowan, M. J., \& Davis, N. O. (2004). Assessment of young children's social-emotional development and psychopathology: Recent advances and recommendations for practice. Journal of Child Psychology and Psychiatry, 45 (1), 109-134

Cashmore, J. (2006). Ethical issues concerning consent in obtaining children's reports on their experience of violence. Child Abuse EN Neglect, 30 (9), 969977.

Chaffin, M. (2000). What types of mental health treatment should be considered for maltreated children? In H. Dubowitz \& D. DePanfilis (eds), Handbook for child protection practice. Thousand Oaks, CA: Sage.

Cohen, J. A., Mannarino, A. P., Kliethermes, M., \& Murray, L.A. (2012). Trauma-focused CBT for youth with complex trauma. Child Abuse and Neglect, 36 (6), 528-541.

Collin-Vézina, D., Coleman, K., Milne, L., Sell, J., \& Daigneault, I. (2011). Trauma experiences, maltreatment-related impairments, and resilience among child welfare youth in residential care. International Journal of Mental Health $\mathfrak{F}$ Addiction, 9 (5), 577-589. 
Conradi, L, Agosti, J., Tullberg, E., Richardson, L., Langan, H., Ko, S., \& Wilson, C. (2011). Promising practices and strategies for using traumainformed child welfare practice to improve foster care placement stability: A breakthrough series collaborative. Child Welfare, 90 (6), 207-225.

Cook, A., Spinazzola, J., Ford, J., Lanktree, C., Blaustein, M., Cloitre, M., DeRosa, R., Hubbart, R., Kagan, R., Liautaud, J., Mallah, K., Olafson, E., $\&$ van der Kolk, B. (2005). Complex trauma in children and adolescents. Psychiatric Annals, 35 (5), 390-398.

Cooley, M. E., \& Petren, R. E. (2011). Foster parent perceptions of competency: Implications for foster parent training. Children and Youth Services Review, 33 (10), 1968-1974.

Connor, D. F., Doerfler, L. A., Toscano, P. F., Volungis, A. M., \& Steingard, R. J. (2004). Characteristics of children and adolescents admitted to a residential treatment center. Journal of Child and Family Studies, 13 (4), 497-510.

Cyr, K., Chamberland, C., Lessard, G., Clement, M-E., Wemmers, J.-A., CollinVézina, D., Gagné, M-H., \& Damant, D. (2012). Polyvictimization in a child welfare sample of children and youths. Psychology of Violence, 2 (4), 385-400.

Cyr, K., Chamberland, C., Clément, M.-È., Lessard, G., Gagné, M.-H., CollinVézina, D., Wemmers, J.-A., \& Damant, D. (2013). Exposure to violence, victimization and multiple victimizations of children and youth in the population of Québec. Child Abuse \& Neglect, 37, 814-820.

Dale, N., Baker, A. J. L., Anastasio, E., \& Purcell, J. (2007). Characteristics of children in residential treatment in New York State. Child Welfare: Journal of Policy, Practice, and Program, 86 (1), 5-27.

D’Andrea, W., Ford, J., Stolbach, B., Spinazolla, J., \& van der Kolk, B.A. (2012). Understanding interpersonal trauma in children: Why we need a developmentally appropriate trauma diagnosis. American Journal of Orthopsychiatry, 82 (2), 187-200.

Dilley, J. B., Weiner, D. A., Lyons, J. S., \& Martinovich, Z. (2007). The validity of the Child and Adolescent Needs and Strengths Assessment. Online submission: Eric Educational Resources Center.

Dorsey, S., Burns, B. J., Southerland, D. G., Cox, J. R., Wagner, H. R., \& Farmer, E. M. Z. (2012). Prior trauma exposure for youth in treatment foster care. Journal of Child and Family Studies, 21 (5), 816-824.

Doyle, A., Ostrander, R., Skare, S., Crosby, R. D., \& August, G. J. (1997). Convergent and criterion related validity of the Behavior Assessment System for Children-Parent Rating Scale. Journal of Clinical Child Psychology, 26 (3), 276-284.

Egelund, T., \& Lausten, M. (2009). Prevalence of mental health problems among children placed in out-of-home care in Denmark. Child $\mathfrak{O}$ Family Social Work, 14 (2), 156-165.

Farrington, A., Waller, G., Smerden, J., \& Faupel, A. W. (2001). The Adolescent Dissociative Experience Scale: Psychometric properties and differences in scores across age groups. Journal of Nervous and Mental Disorders, 189 (10), 722-727. 
Finkelhor, D., Ormrod, R. K., Turner, H. A., e\& Hamby, S. L. (2005). Measuring poly-victimization using the Juvenile Victimization Questionnaire. Child Abuse or Neglect, 29 (11), 1297-1312.

Finkelhor, D., Ormrod, R. K., \& Turner, H. A. (2007). Poly-victimization and trauma in a national longitudinal cohort. Development and Psychopathology, 19 (1), 149-166.

Fletcher, K. E. (1996a). Psychometric review of Dimensions of Stressful Events (DOSE) Rating Scale. In B. H. Stamm (ed.), Measurement of stress, trauma, and adaptation. Lutherville, MD: Sidran Press.

Fletcher, K. E. (1996b). Measuring school-aged children's PTSD: Preliminary psychometrics of four new measures. Paper presented at the Twelfth Annual Meeting of the International Society for Traumatic Stress Studies, San Francisco.

Fletcher, K. E. (2007). Posttraumatic stress disorder. In E. J. Mash \& R.A. Barkley (eds.), Assessment of childbood dis- orders (4th ed., 398-486). New York, NY: Guilford Press.

Fletcher, K. E., Spilsbury, J. C., Creeden, R., \& Friedman, S. (2006). Psychometrics of the Dimensions of Stressful Events (DOSE) scale for children exposed to violence. Poster presentation, 22nd Annual Conference for the International Society for Traumatic Stress Studies, Hollywood, CA.

Ford, J., Ghosh Ippen, C., Wolpaw, J., Racusin, R., Rogers, K., Ellis, C., Schiffman, J., Ribbe, D., Cone, P., Lukovitz, M., \& Edwards, J. (2005). Traumatic Events Screening Inventory for Children (TESI-C) Version 8.5. Dartmouth, VT: National Centre for PTSD and Dartmouth Child Psychiatry Research Group.

Forde, D. R., Baron, S. W., Scher, C. D., \& Stein, M. B. (2012). Factor Structure and reliability of the Childhood Trauma Questionnaire and prevalence estimates of trauma for male and female street youth. Journal of Interpersonal Violence, 27 (2), 364-379.

Frick, P. J., Barry, C. T., \& Kamphaus, R. W. (2009). Chapter 6: Self-report inventories. In Clinical assessment of child and adolescent personality and behavior ( $3^{\text {rd }}$ ed.).

Garrusi, B. \& Nakhaee, N. (2009). Validity and reliability of a Persian version of the Childhood Trauma Questionnaire Psychological Reports, 104 (2), 509516.

Ghosh Ippen, C., Ford, J., Racusin, R., Acker, M., Bosquet, K., Rogers, C., \& Edwards, J. (2002). Trauma events screening inventory-parent report revised. San Francisco: The Child Trauma Research Project of the Early Trauma Network and The National Center for PTSD Dartmouth Child Trauma Research Group.

Glisson, C., \& Green, P. (2011). Organizational climate, services, and outcomes in child welfare systems. Child Abuse and Neglect, 35 (8), 582-591.

Goodman, R. (1997). The Strengths and Difficulties Questionnaire: A research note. Journal of Child Psychology o Psychiatry $\mathfrak{F}$ Allied Disciplines, 38 (5), 581-586. 
Greenwald, R. (1999). Child trauma measures for research and practice. Poster session presented at the annual meeting of the EMDR International Association, Las Vegas. Retrieved from http://www.childtrauma.com.mezpost. html.

Griffin, A. K., Ingram, S. D., Barth, R. P., Trout, A.L., Doppong Hurley, K., Thompson, R. W., \& Epstein, M. H. (2009). The family characteristics of youth entering a residential care program. Residential Treatment for Children $\sim$ Youth, 26 (2), 135-150.

Grills, A. E., \& Ollendick, T. H. (2002). Issues in parent-child agreement: The case of structured diagnostic interviews. Clinical Child and Family Psychology Review, 5 (1), 57-83.

Guibord, M., Bell, T., Romano, E., \& Rouillard, L. (2011). Risk and protective factors for depression and substance abuse in an adolescent child welfare sample. Children and Youth Services Review, 33 (11), 2127-2137.

Hamby, S. L., \& Finkelhor, D. (2000). The victimization of children: Recommendations for assessment and instrument development. Journal of the American Academy of Child $\mathcal{G}$ Adolescent Psychiatry, 39 (7), 829-840.

Hamby, S. L., Finkelhor, D., Ormrod, R. K., \& Turner, H. A. (2004). The Comprehensive Juvenile Victimization Questionnaire. Durham, $\mathrm{NH}$ : University of New Hampshire.

Hélie, S., Poirier, M.-A., et Turcotte, D. (soumis). Risk of maltreatment recurrence after exiting substitute care. Child Maltreatment.

Hovens, J. E., Van der Ploeg, H. M., Klaarenbeek, M. T. A., Bramsen, I., Schreuder, J. N., \& Rivero, V. V. (1994). The assessment of posttraumatic stress disorder with the Clinician Administered PTSD Scale: Dutch results. Journal of Clinical Psychology, 50 (3), 325-340.

Hummer, V. L., Dollard, N., Robst, J., \& Armstrong, M. I. (2010). Innovations in implementation of trauma-informed care practices in youth residential Treatment: A curriculum for organizational change. Child Welfare, 89 (2), 79-95.

Hussey, D. L., \& Guo, S. (2002). Profile characteristics and behavioral change trajectories of young residential children. Journal of Child and Family Studies, 11 (4), 410-410.

Igelman, R., Taylor, N., Gilbert, A., Ryan, B., Setinberg, A., Wilson, C., \& Mann, G. (2007). Creating More Trauma-Informed Services for Children using Assessment-Focused Tools. Child Welfare League of America, 18 pp.

Ivanova, M. Y., Achenbach, T. M., Dumenci, L., Rescorla, L. A., Almqvist, F., Weintraub, S., Bilenberg, N., Bird, H., Chen, W. J., Dobrean, A., Döpfner, M., Erol, N., Fombonne, E., Castro Fonseca, A., Frigerio, A., Grietens, H., Hannesdottir, H., Kanbayashi, Y., Lambert, M., Larsson, B., Leung, P., Liu, X., Minaei, A., Mulatu, M. S., Novik, T. S., Ja Oh, K., Roussos, A., Sawyer, M., Simsek, Z., Steinhausen, H.-C., Winkler Metzke, C., Wolanczyk, T., Jang, H.-J., Zilber, N., Zukauskiene, R. \& Verhulst, F. C. (2007). Testing the 8-syndrome structure of the Child Behavior Checklist in 30 societies. Journal of Clinical Child or Adolescent Psychology, 36 (3), 405-417. 
Jannsens, A., \& Deboutte, D. (2009). Screening for psychopathology in child welfare: The Strengths and Difficulties Questionnaire (SDQ) compared with the Achenbach System of Empirically Based Assessment (ASEBA). European Child and Adolescent Psychiatry, 18 (11), 691-700.

Kinniburgh, K., Blaustein, M., Spinazzola, J., \& van der Kolk, B. (2005). Attachment, self-regulation, and competency: A comprehensive intervention framework for children with complex trauma. Psychiatric Annals, 35 (5), 424-430.

Kolko, D., Hurlburt, M., Zhang, J., Barth, R., Leslie, L., \& Burns, B. (2010). Posttraumatic stress symptoms in children and adolescents referred for child welfare investigation: A national sample of in-home and out-of-home care. Child Maltreatment, 15 (1), 48-53.

Lafortune, D. (2006). Médicaments psychotropes et placement des jeunes en centres de réadaptation. In J. Collin, L. Monnais \& M. Otero (eds.), Le médicament au coeur de la socialité contemporaine (223-245). Québec: Presses de l'Université du Québec.

Lanktree, C. B., Gilbert, A. M., Briere, J., Taylor, N., Chen, K., Maida, C. A., \& Saltzman, W. R. (2008). Multi-informant assessment of maltreated children: Convergent and discriminant validity of the TSCC and TSCYC. Child Abuse $\mathfrak{*}$ Neglect, 32 (6), 621-625.

Lyons, J. S. (2004). Redressing the emperor: Improving the children's public mental bealth system. Westport, CT: Praeger.

Lyons, J. S., Griffin, E., Fazio, M., \& Lyons, M. B. (1999). Child and Adolescent Needs and Strengths: An information integration tool for children and adolescents with mental health challenges (CANS-MH), manual. Chicago, IL: Buddin Praed Foundation.

Lyons, J. S., Small, L., Weiner, D. A., \& Kisiel, C. (2008). Child and Adolescent Needs and Strengths: Illinois Department of Children and Family Services (Version 2.0). Chicago, IL: Praed Foundation.

Lyons, J. S., Uziel-Miller, N., Reyes, F., \& Sokol, P. T. (2000). The prevalence of strengths and their association with psychopathology and functioning for children and youth in residential treatment. Journal of the American Academy of Child and Adolescent Psychiatry, 39 (2), 176-181.

Margolin, G. (2000). The effects of family and community violence on children. Annual Review of Psychology, 51, 1-40.

Mash, E., \& Hunsley, J. (2005). Developing guidelines for the evidence-based assessment of child and adolescent disorders: Issues and challenges. Journal of Clinical Child and Adolescent Psychology, 34 (3), 362-379.

Mathews, B. \& Kenny, M. C. (2008). Mandatory reporting legislation in the United States, Canada, and Australia: A cross-jurisdictional review of key features, differences, and issues. Child Maltreatment, 13 (1), 50-63.

McMillen, J. C., Zima, B. T., Scott, L. D., Auslander, W. F., Munson, M. R., Ollie, M. T., \& Spitznagel, E. L. (2005). Prevalence of psychiatric disorders among older youths in the foster care system. Journal of the American Academy of Child and Adolescent Psychiatry, 44 (1), 88-95. 
Milne, L., \& Collin-Vézina, D. (sous presse). Developing a portrait of sexually abused youth placed in residential care: A multiple source comparison. Journal of Child Sexual Abuse.

Mueser, K., Yarnold, P. R., Rosenberg, S., Swett, C., Miles, K., \& Hill, D. (2000). Substance use disorder in hospitalized severely mentally ill psychiatric patients: Prevalence, correlates, and subgroups. Schizophrenia Bulletin, 26 (1), 179-192.

Muris, P., Mereckelbach, H., \& Peeters, E. (2003). The Links between the Adolescent Dissociative Experiences Scale (A-DES), Fantasy Proneness, and Anxiety Symptoms. Journal of Mental and Nervous Disease, 191 (1), 18-24.

Nader, K. (2008). Understanding and assessing trauma in children and adolescents: Measures, methods, and youth in context. New York, NY: Routledge Taylor Francis Group.

Nader, K., Kriegler, J. A., Blake, D. D., Pynoos, R. S., Newman, E., \& Weathers, F. W. (1996). Clinician Administered PTSD Scale, Child and Adolescent Version. White River Junction, VT: National Center for PTSD.

National Child Traumatic Stress Network (2008). Child Welfare Trauma Training Toolkit: Trauma Referral Tool. Retrieved from: www.nctsnet.org/ nctsn_assets/pdfs/CWT3_SHO_Referral.pdf

Ohan, J., Myers, K., \& Collett, B. (2002). Ten-year review of rating scale IV: Scales assessing trauma and its effects. Academy Child Adolescent Psychiatry, 41 (12), 1401-1422.

Paivio, S. C., \& Cramer, K. M. (2004). Factor structure and reliability of the Childhood Trauma Questionnaire in a Canadian undergraduate student sample. Child Abuse Er Neglect, 28 (8), 889-904.

Petrenko, C. L., Culhane, S. E., Garrido, E. F., \& Taussig, H. N. (2011). Do youth in out-of-home care receive recommended mental health and educational services following screening evaluations? Children and Youth Services Review, 33 (10), 1911-1918.

Pynoos, R., Rodriguez, N., Steinberg, A., Stuber, M., \& Frederick, C. (1998). The UCLA PTSD reaction index for DSM IV (Revision 1). Los Angeles, CA: UCLA Trauma Psychiatry Program.

Reynolds, C. R., \& Kamphaus, R. W. (2004). Behavior Assessment System for Children-Second Edition manual. Circle Pines, MN: American Guidance Service Publishing.

Ribbe, D. (1996). Psychometric review of Traumatic Events Screening Inventory for Children (TESI-C). In B. H. Stamm (Ed.), Measurement of stress, trauma, and adaptation. Lutherville, MD: Sidran Press.

Rodriguez, N., Steinberg, A. S., Saltzman, W. S., \& Pynoos, R. S. (2001). PTSD Index: Psychometric analyses of the adolescent version. Symposium conducted at the Annual Meeting of the International Society for Traumatic Stress Studies, New Orleans, LA.

Roussos, A., Goenjian, A. K., Steinberg, A. M., Sotiropoulou, C., Kakaki, M., Kabakos, C., Karagianni, S., \& Manouras, V. (2005). Posttraumatic stress and depressive reactions among children and adolescents after the 1999 
earthquake in Ano Liosia, Greece. American Journal of Psychiatry, 162 (3), 530-537.

Runtz, M., \& Briere, J. (1986). Adolescent "acting out" and childhood history of sexual abuse. Journal of Interpersonal Violence, 1 (3), 326-333.

Scher, C. D., Stein, M. B., Asmundson, G. J. G., McCreary, D. R., \& Forde, D. (2001). The Childhood Trauma Questionnaire in a community sample: Psychometric properties and normative data. Journal of Traumatic Stress, 14 (4), 843-857.

Sieracki, J. H., Leon, S. C., Miller, S. A., \& Lyon, J. S. (2008). Individual and provider effects on mental health outcomes in child welfare: A three level growth curve approach. Children $\mathfrak{F}$ Youth Services Review, 30 (7), 800-808.

Singer, M. I., Anglin, T. M., Song, L. Y., \& Lunghofer, L. (1995). Adolescent's exposure to violence and associated symptoms of psychological trauma. Journal of the American Medical Association, 273 (6), 477-482.

Smith, S. R., \& Carlson, E. B. (1996). Reliability and validity of the Adolescent Dissociative Experiences Scale. Dissociation: Progress in the Dissociative Disorders, 9 (2), 125-129.

Spilsbury, J. C., Fletcher, K. E., Creeden, R., \& Friedman, S. (2008). Psychometric properties of the dimensions of Stressful Events Rating Scale. Traumatology, 14 (4), 1-16.

Steele, W., \& Malchiodi, C. A. (2012). Trauma-Informed Practices with Children and Adolescents. New York, NY: Taylor and Francis Group, LLC.

Stevenson, J. (1999). The treatment of the long-term sequelae of child abuse. Journal of Child Psychology and Psychiatry, 40 (1), 89-111.

Stoltzfus, E. (2011). Child Welfare: Recent and proposed federal funding. Congressional Research Service report for Congress, RL34121, 22 pp. http:// greenbook.waysandmeans.house.gov/sites/greenbook.waysandmeans. house.gov/files/2011/images/RL34121_gb.pdf

Strand, V. C., Sarmiento, T. L., \& Pasquale, L. E. (2005). Assessment and screening tools for trauma in children and adolescents: A review. Trauma, Violence, $\mathfrak{O}$ Abuse, 6 (1), 55-78.

Tan, C. S. (2007). Test review: Reynolds, C. R., \& Kamphaus, R. W. (2004). Behavior Assessment System for Children (2nd ed.). Assessment for Effective Intrvention, 32, 121-124.

Tarren-Sweeney, M. (2008). The mental health of children in out-of-home care. Current Opinion in Psychiatry, 21 (4), 345-349.

Tarren-Sweeney, M. (2007). The Assessment Checklist for Children - ACC: A behavioral rating scale for children in foster, kinship and residential care. Children and Youth Services Review, 29 (5), 672-691.

Taylor, N., \& Siegfried, C. (2005). Helping children in the child welfare system heal from trauma: A systems integration approach. Los Angeles and Durham: National Child Traumatic Stress Network.

Taylor, N, Steinberg, A., \& Wilson, C. (2006). Child Welfare Trauma Referral Tool. San Diego, CA: Chadwick Center for Children and Families. 
Thombs, B. D., Lewis, C., Bernstein, D. P., Medrano, M. A., \& Hatch, J. P. (2007). An evaluation of the measurement equivalence of the Childhood Trauma Questionnaire-Short Form across gender and race in a sample of drug-abusing adults. Journal of Psychosomatic Research, 63 (4), 391-398.

Trout, A. L., Hagaman, J. L., Chmelka, M. B., Gehringer, R., Epstein, M. H., \& Reid, R. (2008). The academic, behavioral, and mental health status of children and youth at entry to residential care. Residential Treatment for Children $\mathfrak{F}$ Youth, 25 (4), 359-374.

Van der Kolk, B. A., \& Courtois, C. A. (2005). Editorial comments: Complex developmental trauma. Journal of Traumatic Stress, 18 (5), 385-388.

Walter, U. M. (2007). Best Practices in Children's Mental Health-Report \#20: Residential Treatment: A Review of the National Literature. The University of Kansas, $41 \mathrm{pp}$.

Warnick, E. M., Bracken, M. B., \& Kasl, S. (2007). Screening efficiency of the Child Behavior Checklist and Strengths and Difficulties Questionnaire: A systematic review. Child and Adolescent Mental Health, 13 (3), 140-147.

Weathers, F. W., \& Keane, T. M. (2007). The Criterion A problem revisited: Controversies and challenges in defining and measuring psychological trauma. Journal of Traumatic Stress, 20 (2), 107-121.

Weiner, D. A., Schneider, A., \& Lyons, J.S. (2009). Evidence-based treatments for trauma among culturally diverse foster care youth: Treatment retention and outcomes. Children and Youth Services Review, 31 (11), 1199-1205

Weis, R., \& Smenner, L. (2007). Construct validity of the Behavior Assessment System for Children (BASC) Self-Report of Personality: Evidence from adolescents referred to residential treatment. Journal of Psychoeducational Assessment, 25 (2), 111-126.

Weis, R., Wilson, N. L., \& Whitemarsh, S. M. (2005). Evaluation of a militarystyle residential treatment program for adolescents with academic and conduct problems. Journal of Clinical Child and Adolescent Psychology, 34 (4), 692-705. DOI : 10.1207/s15374424jccp3404_11

Wright, K. D., Asmundson, G. J. G., McCreary, D. R., Scher, C., Hami, S., \& Stein, M. (2001). Factorial validity of the Childhood Trauma Questionnaire in men and women. Depression and Anxiety, 13 (4), 179-183.

ABSTRACT - Considering that youth placed in rehabilitation centres have often experienced multiple traumatic events, and that specialized trauma-focused services are not routinely implemented in child protection services, a standardized assessment of traumatic events and symptoms should be put in place for this at-risk clientele. The purpose of this article is to provide an overview of psychometric measurement tools used to assess trauma in youth. This information is necessary in order to facilitate the development of a solid and cohesive mental health program that is focused on trauma in youth protection. Based on a systematic screening, 13 tools designed to collect information regarding traumatic events and symptoms were selected, most of which are youth self-reports. Practical and methodological considerations of these tools are presented. The implementation of a standardized assessment of trauma for children and youth in rehabilitation centres could serve as a catalyst for the development of 
specialized services for this particularly vulnerable and high needs clientele. The most promising trauma-focused interventions are discussed.

KEYWORDS - Trauma, assessment, rehabilitation centres, youth.

RESUMEN - Teniendo en cuenta que los jóvenes internados en centros de readaptación vivieron frecuentemente eventos traumáticos múltiples, y que los servicios especializados en el trauma no están implantados sistemáticamente en la protección de la minoridad, seria deseable que se estableciera una evaluación estandarizada de los eventos y de las secuelas traumáticas de la clientela en riesgo. El objetivo del presente artículo es ofrecer una síntesis de los instrumentos psicométricos para evaluar el trauma en los jóvenes, y esto, con el objetivo de volver más accesible dicha información, necesaria para desarrollo de un programa sólido y cohesivo de salud mental, centrado sobre el traumatismo en la protección del menor. Sobre una base de un enfoque sistemático, han sido seleccionados 13 instrumentos que apuntan a los acontecimientos y a los sintomas traumáticos; en su mayoría se trata de cuestionarios llenados por los jóvenes y de entrevistas dirigidas. Las consideraciones prácticas y metodológicas de dichos instrumentos son presentados. La implantación de una evaluación sistemática de los niños y adolescentes institucionalizados podría servir a la puesta en marcha de servicios especializados en dicha clientela, para la cual las necesidades urgen. Las intervenciones más prometedoras son discutidas.

PALABRAS-CLAVE - Trauma, evaluación, centros de readaptación, adolescentes. 